\title{
Population Pharmacokinetics and Pharmacodynamics Modelling of Dilmapimod in Severe Trauma Subjects at Risk for Acute Respiratory Distress Syndrome
}

\author{
Shuying Yang ${ }^{1}$ Teodora Pene Dumitrescu ${ }^{2}$
}

Published online: 21 December 2016

(C) The Author(s) 2016. This article is published with open access at Springerlink.com

\begin{abstract}
Introduction Dilmapimod is a potent p38 mitogen-activated protein kinase (MAPK) inhibitor and was investigated in a study (NCT00996840) for its anti-inflammatory effect in non-head injury trauma patients at risk for developing acute respiratory distress syndrome (ARDS). The purpose of this paper is to present the details of the development of a population pharmacokinetic (PK) model, an empirical population placebo response model, and the exploration of a PK/pharmacodynamic (PD) model of dilmapimod.

Methods A population PK model was developed to characterise the PK profile of dilmapimod in this patient population; the potential effect of available covariates on the PK of dilmapimod was evaluated. An empirical population placebo response model was conducted, and a population PK/PD model was explored to evaluate the relationship between dilmapimod concentration and C-reactive protein (CRP) (a systemic biomarker of p38 inhibition). All analyses were performed using NONMEM software.

Results Following intravenous dosing, dilmapimod was quickly distributed to peripheral compartments and then slowly eliminated. The plasma concentration of dilmapimod was adequately described by a three-compartment model. It increased approximately proportionally to the
\end{abstract}

increase in dose. The population clearance (CL) parameter value was $35.87 \mathrm{~L} / \mathrm{h}$, and the steady-state volume of distribution (Vss) [sum of the volume of distribution of the central compartment $(\mathrm{Vc})$ and of the peripheral compartments V2 and V3] was $160 \mathrm{~L}$. The effect of body mass index (BMI) on CL and inter-compartment clearance (Q2) was found statistically significant, with an increase in BMI of $1 \mathrm{~kg} / \mathrm{m}^{2}$ resulting in a $1.79 \mathrm{~L} / \mathrm{h}$ and $0.52 \mathrm{~L} / \mathrm{h}$ increase in $\mathrm{CL}$ and Q2, respectively. The CRP profile post injury was adequately described by an indirect response model, with a sharp increase in the CRP levels following injury, followed by them slowly diminishing. Data exploration indicated potential drug effects of dilmapimod on inhibiting the production of CRP levels; however, the current small dataset did not show a statistically significant improvement in the PK/PD modelling.

Conclusion The population PK modelling adequately evaluated the dilmapimod plasma concentration-time profiles in severe trauma subjects at risk for ARDS, and BMI was found to be a significant covariate in the PK model. An indirect response model was adequate to describe the production and degradation of CRP levels in these subjects.
Shuying Yang

shuying.y.yang@gsk.com

1 Clinical Pharmacology Modelling and Simulation, RD Projects Clinical Platforms and Sciences, GlaxoSmithKline, Stockley Park West, 1-3 Ironbridge Road, Uxbridge, Middlesex UB11 1BT, UK

2 Clinical Pharmacology Modeling and Simulation, GlaxoSmithKline, Research Triangle Park, NC, USA 


\section{Key Points}

Dilmapimod plasma concentration-time profiles in severe trauma subjects at risk for acute respiratory distress syndrome were adequately described by a three-compartment model. Following intravenous dosing, dilmapimod was quickly distributed to peripheral compartments and then slowly eliminated in a multi-exponential manner. The plasma concentration of dilmapimod increased approximately proportionally to the increase in dose. Body mass index was a significant covariate in the pharmacokinetic model.

The C-reactive protein (CRP) profile post injury was adequately described by an indirect response model, with a sharp increase in the CRP levels following injury, followed by them slowly diminishing. There was a trend in dilmapimod inhibiting the production of CRP; the current small dataset did not show a statistically significant improvement in the pharmacokinetic/pharmacodynamic modelling.

\section{Introduction}

Acute respiratory distress syndrome (ARDS) is a lifethreatening complex condition associated with severe hypoxia and respiratory failure. Despite improved understanding of the pathogenesis of the disease, there is no effective pharmacological treatment for ARDS [1, 2].

Severe trauma has been recognised as a potential cause of ARDS due to indirect lung injury [3]. Tissue injury following trauma is mediated by multiple immunological processes involving neutrophils, macrophages, and dendritic cells, leading to elevated levels of circulating proinflammatory cytokines such as interleukin 6 (IL-6), tumour necrosis factor alpha (TNF- $\alpha$ ), and IL-8, both in the lungs and in systemic circulation [1]. Therefore, attenuation of pro-inflammatory cytokine signalling may be beneficial in both treatment and prevention of ARDS.

The p38 mitogen-activated protein kinase (MAPK) is a serine-threonine protein kinase that phosphorylates intracellular signal transduction molecules involved in the regulation of inflammatory cytokine biosynthesis [4-7]. It is believed that activation of the p38 MAPK pathway is involved in the mechanism of ARDS [8, 9]; therefore, inhibition of the p38 MAPK signalling pathway at the early stage of ARDS may lead to decreased expression of proinflammatory cytokines known to be involved in the pathophysiology of ARDS.
Dilmapimod is a potent inhibitor of the p38 MAPK pathway. Multiple studies have evaluated and shown its pharmacological effect in inhibiting production of cytokines in different disease populations following oral administration [4-7]. However, no systemic pharmacokinetic (PK) data were reported in those studies.

The study we report on in this paper was conducted to investigate the hypothesis of p38 inhibition in the early stage of ARDS, and to evaluate the safety, tolerability, PK and pharmacodynamics (PD) of dilmapimod following intravenous administration in non-head injury trauma patients at risk for developing ARDS. The study objectives, endpoints, inclusion and exclusion criteria, as well as the primary results have been described elsewhere [10].

The aim of this paper is to present the details of the development of a population PK model, an empirical placebo response model, and the exploration of a population PK/PD model of dilmapimod. Specifically, the following analyses were conducted: (1) a population PK model was developed to characterise the PK profile of dilmapimod in this patient population; (2) the potential effect of available covariates on the PK of dilmapimod was evaluated; and (3) a population PK/PD model was conducted to evaluate the relationship between dilmapimod concentration and C-reactive protein (CRP), a systemic biomarker of p38 inhibition, which displayed reduced plasma levels compared with placebo [10].

\section{Methods}

\subsection{Study Design}

A phase IIa, randomised, double-blind, placebo-controlled, parallel-group study was conducted at six sites in the USA to evaluate the safety, tolerability, and systemic PK and PD profiles of dilmapimod compared with placebo in patients with major trauma (exclusive of severe head injury) and at risk for developing ARDS (clinicaltrial.gov NCT00996840). Details of the study's design are provided in Christie et al. [10]. Briefly, eligible subjects were randomised into the trial within the first 24-26 h after known trauma had occurred. Four cohorts were recruited and dosed for 3 consecutive days via an intravenous infusion. Cohort 1 patients were randomised 3:1 to receive 3 days of dilmapimod (3 mg intravenously) infused over $4 \mathrm{~h}$ or placebo; cohort 2 patients were randomised $2: 1$ to receive 3 days of dilmapimod ( $7.5 \mathrm{mg}$ intravenously) infused over 24 h or placebo; cohort 3 patients were randomised $3: 1$ to receive 3 days of dilmapimod ( $7.5 \mathrm{mg}$ intravenously) infused over $4 \mathrm{~h}$ or placebo; and cohort four patients were randomised $3: 1$ to receive 3 days of dilmapimod $(10 \mathrm{mg}$ intravenously) infused over $24 \mathrm{~h}$ or placebo. A follow-up 
visit was conducted on day 7, i.e. 4 days after intravenous dosing had finished, or at discharge from the hospital if earlier than day 7 (but no earlier than $12 \mathrm{~h}$ after dosing had been completed).

Blood samples for PK analysis were collected at the following times:

- For cohorts 1 and 3 (4 h infusion): on day 1, pre-dose (before the first dose), then 4, 4.25, 5, 8, and $12 \mathrm{~h}$ after the first dose; pre-dose on day 2 (before the second dose) and day 3 (before the third dose); then $24 \mathrm{~h}$ after the third dose.

- For cohorts 2 and 4 (24 h infusion): pre-dose on day 1 (before the first dose) and day 2 (before the second dose); on day 3, pre-dose (before the third dose), then at $24 \mathrm{~h}$ (immediately before the infusion stopped), $24 \mathrm{~h}$ $10 \mathrm{~min}$ (10 min after the third dose), $24 \mathrm{~h} 45 \mathrm{~min}$

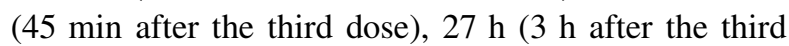

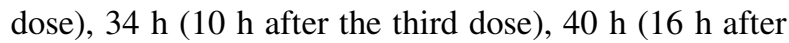
the third dose), and $48 \mathrm{~h}$ ( $24 \mathrm{~h}$ after the third dose).

Blood samples for the PD analysis for all four cohorts were collected at the following times:

- Day 1: pre-dose and 6, 12, and $18 \mathrm{~h}$ after the first dose.

- Day 2: pre-dose.

- Day 3: pre-dose and 24 and $48 \mathrm{~h}$ after the third dose.

\subsection{Analytical Method for PK Analysis}

Human plasma samples were analysed for dilmapimod (SB-681323) using a validated analytical method based on protein precipitation, followed by high-performance liquid chromatography-tandem mass spectrometry (HPLC-MS/ MS) analysis. The lower limit of quantification for dilmapimod (SB-681323) was $0.1 \mathrm{ng} / \mathrm{mL}$ using a $50 \mu \mathrm{L}$ aliquot of human plasma and the higher limit of quantification was $100 \mathrm{ng} / \mathrm{mL}$.

Quality control samples (QC), prepared at three different analyte concentrations and stored with study samples, were included with each batch of samples along with separately prepared calibration standards. For the analysis to be acceptable, no more than one-third of the total QC results and no more than one-half of the results from each concentration level were to deviate from the nominal concentration by more than $15 \%$. The applicable analytical runs met all predefined run acceptance criteria.

\subsection{Analytical Method for the PD Analysis}

Human serum CRP levels were quantified by an immunoturbidimetric assay (CRP Latex, Beckman Coulter, Pasadena, California, USA), in which CRP reacts specifically with anti-human CRP antibodies coated on latex particles to yield insoluble aggregates. The absorbance of these aggregates is proportional to the CRP concentration in the sample. The assay linear range for CRP was from 1.0 to $480 \mathrm{mg} / \mathrm{L}$.

\subsection{Datasets}

\subsubsection{PK Dataset}

A total of 471 dilmapimod concentration records from 57 subjects were available for inclusion in the PK analysis dataset. Two outlier subjects (one from each of the 24-h infusion groups; a total of 14 samples) with a concentration range over the sampling interval over ten times greater than the range observed in the majority of the subjects were excluded from the model. Inclusion of these subjects resulted in failure of minimisation. In addition, all postdose concentrations (seven samples) on day 3 and onward from a subject in the 24-h 10-mg intravenous infusion group were excluded because of missing infusion volume. Furthermore, 19 samples at planned pre-dose time points were excluded because their value was consistent with being drawn after the start of infusion (sudden jump in concentration at pre-dose following a decline in concentrations at the previous time points). In total, 40 concentration records $(8.5 \%)$ were excluded. Ten $(2.1 \%)$ of the concentrations were not quantifiable (NQ). All NQ values were set to missing.

\subsubsection{CRP Dataset}

To assemble the PK/PD analysis dataset for CRP, the final PK model was used to predict dilmapimod concentrations at the times where the CRP biomarker was measured for each patient in the dataset. The predicted concentrations were merged into the CRP biomarker dataset and used in the PK/PD analysis. The patients excluded from the PK/PD analysis were the patients who had only dosing records in the PK dataset, the patients excluded from the PK analysis dataset because they had outlier profiles, and one additional patient who had missing CRP measurements. Due to the fact that no measurement of CRP was available for the time of injury, the baseline CRP concentration at the time of injury was assumed to be the CRP level for healthy individuals $(1.35 \mathrm{mg} / \mathrm{L}$, with added $10 \%$ variability to incorporate the uncertainty) [6, 11-15] (see Sect. 2.5.2.1 for more details). The final PK/PD analysis dataset comprised a total of 651 dilmapimod and CRP records (478 from subjects on active treatment and 173 from subjects on placebo) from 73 subjects (53 subjects on active treatment and 20 placebo subjects). 


\subsection{Methodology}

The first-order conditional estimation method with interaction (FOCEI) was used for all PK and PK/PD analyses. The analyses were conducted with NONMEM (version 7.1.2.) (ICON Development Solutions; Ellicott City, MD, USA).

\subsubsection{Population PK Modelling}

Population PK modelling for dilmapimod was developed using non-linear mixed-effects analyses. With $y_{\mathrm{ij}}$ indicating the plasma concentration of the $i$ th subject measured at time $t_{\mathrm{j}}$, the nonlinear mixed effect model was described as:

$y_{i j}=f\left(\theta_{i}, D_{i}, t_{j}\right) \times \exp \left(\varepsilon_{i j}\right)$

where $f$ represents the structural PK model, $\theta_{i}$ indicates the PK parameters in the model for subject $i, D_{i}$ is the dose administered for subject $i, t_{\mathrm{j}}$ is the time of the plasma concentration measurement, and $\varepsilon_{i j}$ is the residual error of the measurement, and is assumed to follow normal distribution with a mean of 0 and variance of $\sigma^{2}$.

Exploratory analysis of dilmapimod plasma concentration data in healthy subjects indicated that the best structural model to describe the dilmapimod PK was a threecompartment model with first order elimination. The model structure is shown in Fig. 1 (left panel). This model was used for modelling the PK profile of dilmapimod in an acute lung injury population. A two-compartment model was also tested, but a three-compartment model described the data better.

For a three-compartment model, $\theta_{i}=\left(\mathrm{CL}_{i}, \mathrm{Vc} c_{i}, \mathrm{~V} 2_{i}\right.$, $\mathrm{V} 3_{i}, \mathrm{Q} 1_{i}, \mathrm{Q} 2_{i}$ ), where $\mathrm{CL}$ is the population clearance, $\mathrm{Vc}$, $\mathrm{V} 2$, and V3 are the volumes of distribution for the central compartment and the peripheral compartments, and Q1 and
Q2 are inter-compartment clearance values. All PK parameters were described by population mean $\left(\theta_{\mathrm{pop}, k}\right)$ $(k=1,2, \ldots, 6)$ and a shift of the parameters for the $i$ th subject $\left(\mathrm{ETA}_{i k}\right)$ representing the inter-individual variability, as shown below:

$\theta_{i k}=\exp \left(\theta_{\mathrm{pop}, k}+\mathrm{ETA}_{i k}\right)$

ETA $_{i k}$ was assumed to follow normal distribution with a mean of 0 and a variance-covariance matrix of $\Omega_{\mathrm{k}}$.

\subsubsection{Population PK/PD Modelling}

2.5.2.1 Base CRP Model An indirect response model, shown in Fig. 1 (right panel) and Eq. (3), was applied to describe the systemic CRP levels, where $A(t)$ indicates the amount of CRP in the system, $K_{\text {in }}(t)$ and $K_{\text {out }}(t)$ represent the production rate constant and the degradation rate of CRP, respectively.

$\frac{\mathrm{d} A(t)}{\mathrm{d} t}=K_{\text {in }}(t)-K_{\text {out }}(t) * A(t)$

Based on the current understanding of the mechanism of p38 pathways and the hypothesis for p38 inhibitors, it was assumed that following injury (e.g. trauma), the p38 pathway was activated and therefore led to the production of various cytokines and CRP. Although it is not well known how CRP levels change over time after injury, some literature data have indicated that the CRP level quickly increases after injury in a few hours, reaches peak and then gradually decreases $[6,12-15]$. The following mathematical function for $K_{\text {in }}(t)$ was used to model the change in CRP levels over time following the placebo treatment:

$K_{\text {in }}(t)=K_{\text {in }} 0 * \exp \left(-K_{\text {decline }} * \mathrm{t}\right)$

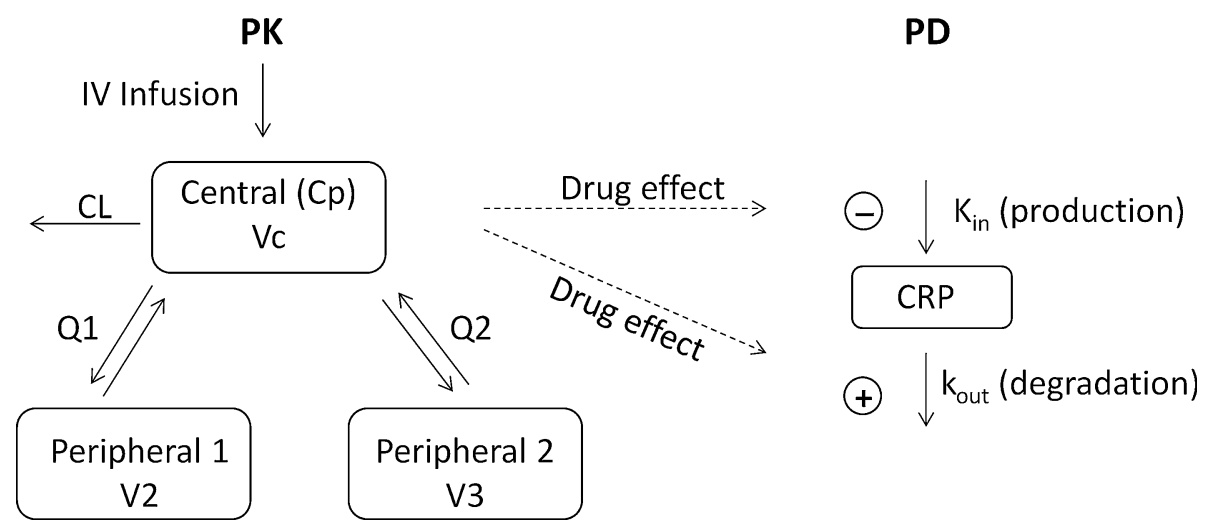

Fig. 1 Pharmacokinetic/pharmacodynamic (PK/PD) model for dilmapimod and C-reactive protein (CRP). Dilmapimod PK were described by a three-compartment model with first-order elimination rates. The relationship between dilmapimod and CRP levels was described using an indirect model with inhibition of CRP input. CL clearance, $C p$ model-predicted dilmapimod concentration, $I V$ intravenous, $K_{\text {in }}$ zero-order production rate constant, $K_{\text {out }}$ first-order elimination rate constant, $Q 1$ and $Q 2$ inter-compartmental clearance, $V c$ volume of distribution of the central compartment, $V 2$ and $V 3$ volume of distribution of the peripheral compartments 
where $K_{\text {in }} 0$ was the CRP production rate immediately following injury and $\exp \left(-K_{\text {decline }}{ }^{*} t\right)$ was used to describe the potential decline in production rate over time in order to correspond to the hypothesis on the general profile of CRP levels after injury without investigational drug.

It is noted that in order to solve the above differential equation, $A(0)$ is required. For this case, as there was no measurement of CRP at time 0 (time of injury), $A(0)$ was set at the level of the corresponding CRP for healthy subjects. For this work, $A(0)=1.35 \mathrm{mg} / \mathrm{L}$ (with added $10 \%$ variability to incorporate the uncertainty) for CRP [11].

2.5.2.2 Drug-Effect Models A brief synopsis of the numerous drug-effect models explored is presented in Table 1. Under the assumption that inhibition of $\mathrm{p} 38$ pathways by the p38 inhibitor, dilmapimod, would reduce CRP production, the effect of dilmapimod on CRP was assessed using inhibitory models, where the production rate of CRP was affected by the level of dilmapimod concentration $(\mathrm{Cp})$ or by dilmapimod exposure [area under the concentration-time curve (AUC)] in the plasma (Table 1). In addition, indirect response models reflecting stimulation of $K_{\text {decline }}$ or stimulation of the dissipation or response $\left(K_{\text {out }}\right)$ were also explored (Table 1$)$.

The parameters in the models are as follows: $\theta=\left(K_{\mathrm{in}} 0\right.$, $K_{\text {decline }}, K_{\text {out }}, I_{\max }, \mathrm{IC} 50, \mathrm{EFF}$, and $\left.\gamma\right)$, where $I_{\max }$ represents the maximum fractional (0-1) extent of inhibition, IC50 is the concentration or AUC of dilmapimod that causes $50 \%$ of the maximum effect $\left(I_{\max }\right)$, and $\gamma$ is the Hill coefficient. Inter-individual variability is introduced by allowing all the parameters to be varied for each individual subject by $\theta_{k}=\theta_{k 0} * \exp \left(\eta_{k i}\right)$, where $\theta_{k 0}$ indicates the typical value of the $k$ th parameter in the list, and $\eta_{k i} \sim N\left(0, \Omega_{k}\right)$ represents the inter-subject variability on the $k$ th parameter $(k=1,2$, $3,4,5,6)$.

Additive and proportional error models were considered to explore the residual variability on the CRP measurements.

\subsection{Covariate Selection}

Covariate analysis was performed to explore measurable sources of variability in the models. Covariate effects were considered for all the model parameters in the PK models as well as parameters in the PK/PD models.

Demographics (age, gender, race, body weight, height), clinical or medical status at entry, disease severity [defined by Injury Severity Score (ISS)], creatinine clearance, and liver function parameters (ALT, AST, total bilirubin and albumin) were considered.

For continuous covariates, $\operatorname{TVPK}_{i k}=\exp \left(\theta_{\mathrm{pop}, k}\right)$ $\times\left(\frac{\mathrm{COV}_{i}}{\mathrm{REF}}\right)^{\theta}$, where $\mathrm{TVPK}_{\mathrm{ik}}$ is the typical value of $k$ th PK parameter for individual $i, \mathrm{COV}_{\mathrm{i}}$ is the value of the $i$ th subject covariate, REF is a value approximately the median of the covariate in the population, and $\theta$ indicates the change in typical parameter value as the covariate changes.

For categorical covariates, $\mathrm{TVPK}_{i k}=\exp \left(\theta_{\mathrm{pop}, k}\right)$ for $\mathrm{CAT}_{i}=0$ and $\mathrm{TVPK}_{i k}=\exp \left(\theta_{\mathrm{pop}, k}+\theta\right)$ for $\mathrm{CAT}_{i}=1$, where $\mathrm{CAT}_{\mathrm{i}}$ represents the categorical covariate variable for subject $i$.
Table 1 Indirect response models used to investigate the effect of dilmapimod on CRP

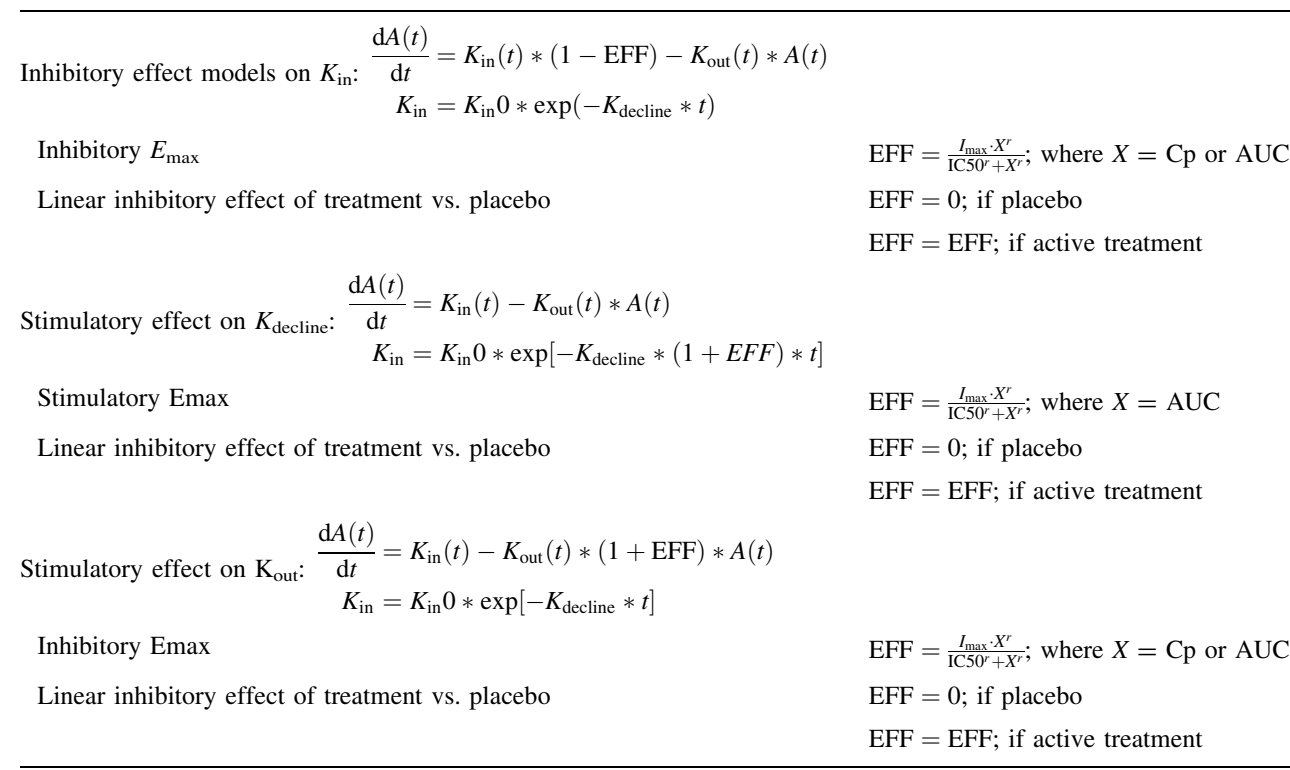

$A U C$ Area under the concentration-time curve, $C p$ model-predicted dilmapimod concentration, $C R P$ C-reactive protein, $E_{\max }$ maximum effect, $E F F$ effect of dilmapimod, $K_{\text {decline }}$ decline in production rate of CRP, $K_{\text {in }}$ production rate constant of CRP, $K_{\text {out }}$ elimination rate constant of CRP 
Potential covariate-parameter relationships were explored graphically using the post hoc random effect values for PK parameters (ETA in NONMEM modelling language) versus each covariate and with simple and multiple regression models using the generalised additive model function (GAM) in Xpose4 (xpose.gam). The selected covariates from these initial analyses were then added to the base model one by one using the forward addition technique, and then removed one at a time using the backward elimination technique.

This forward building technique was continued until all the potential influential covariates were included in the model. The decrease in the objective function $(\Delta)$ was set to $>3.84$ for a single covariate $(p \leq 0.05)$ to be included in the model. If removal of a covariate resulted in an increase in the objective function $(\Delta)$ less than $6.63(p \leq 0.01)$, the covariate under examination was removed from the model. The backward elimination technique continued until all the covariates in the model passed all the criteria.

\subsection{Model Diagnostics and Evaluations}

Uncertainty in parameter estimates was assessed by standard error estimates (returned by NONMEM from the \$COVARIANCE step). Model selection was based on successful minimisation, the objective function value, parameter estimate plausibility, diagnostic plots, shrinkage values, and acceptable relative standard error (RSE). The adequacy of the final model was evaluated using the standard goodness-of-fit method, including plots of population and individual prediction versus observed data, conditional weighted residual versus time, and the visual predictive check method, which was based on 2000 replicates of the original dataset. Model-simulated concentration-time data and associated median and $95 \%$ prediction interval were compared with the observed data for each dilmapimod treatment group (visual predictive check). The $v p c$ command of PsN (version 3.2.4) and xpose.VPC from the XPOSE4 package [12] in R (R Core team 2012) were
Table 2 Parameter estimates of fixed and random effects from the final pharmacokinetic model

\begin{tabular}{|c|c|c|c|c|}
\hline Parameter (unit) & Typical value & $\% \mathrm{RSE}^{\mathrm{a}}$ & Standard error & $95 \% \mathrm{CI}^{\mathrm{b}}$ or $\% \mathrm{CV}^{\mathrm{c}}$ \\
\hline $\mathrm{CL}(\mathrm{L} / \mathrm{h})=\exp \left(\theta_{\mathrm{pop}, 1}\right)$ & 35.9 & & & \\
\hline$\theta_{\text {pop, } 1}$ & 3.58 & 2.25 & 0.0806 & $3.42-3.74$ \\
\hline $\mathrm{Vc}(\mathrm{L})=\exp \left(\theta_{\mathrm{pop}, 2}\right)$ & 8.1 & & & \\
\hline$\theta_{\text {pop, } 2}$ & 2.09 & 24.4 & 0.509 & $1.09-3.09$ \\
\hline $\mathrm{Q} 1(\mathrm{~L} / \mathrm{h})=\exp \left(\theta_{\mathrm{pop}, 3}\right)$ & 28.2 & & & \\
\hline$\theta_{\mathrm{pop}, 3}$ & 3.34 & 10.4 & 0.347 & $2.66-4.02$ \\
\hline $\mathrm{V} 2(\mathrm{~L})=\exp \left(\theta_{\mathrm{pop}, 4}\right)$ & 35.9 & & & \\
\hline$\theta_{\text {pop, } 4}$ & 3.58 & 6.09 & 0.218 & $3.15-4.01$ \\
\hline $\mathrm{Q} 2(\mathrm{~L} / \mathrm{h})=\exp \left(\theta_{\mathrm{pop}, 5}\right)$ & 5.7 & & & \\
\hline$\theta_{\text {pop, } 5}$ & 1.74 & 11.5 & 0.200 & $1.35-2.13$ \\
\hline $\mathrm{V} 3(\mathrm{~L})=\exp \left(\theta_{\mathrm{pop}, 6}\right)$ & 115.6 & & & \\
\hline$\theta_{\text {pop }, 6}$ & 4.75 & 4.90 & 0.233 & $4.29-5.21$ \\
\hline $\mathrm{VSS}(\mathrm{L})=\mathrm{Vc}+\mathrm{V} 2+\mathrm{V} 3$ & 160 & & & \\
\hline Terminal half-life $(h)^{d}$ & 16 & & & \\
\hline BMI covariate on $\mathrm{CL}$ & 1.36 & 18.5 & 0.252 & $0.866-1.85$ \\
\hline BMI covariate on $\mathrm{Q} 2$ & 2.42 & 19.8 & 0.479 & $1.4-3.36$ \\
\hline \multicolumn{5}{|l|}{ Inter-individual variability } \\
\hline $\mathrm{CL}$ & 0.0991 & 23.1 & 0.0229 & 31.5 \\
\hline Q2 & 0.226 & 41.4 & 0.0936 & 47.5 \\
\hline \multicolumn{5}{|l|}{ Residual variability } \\
\hline Sigma & 0.144 & 16.2 & 0.0234 & 37.9 \\
\hline
\end{tabular}

Shrinkage: $5.56 \%$ on CL, $26.5 \%$ on Q2, and $8.06 \%$ on Sigma

Condition number 90.63

$B M I$ Body mass index, $C I$ confidence interval, $C L$ clearance, $C V$ coefficient of variation, $Q 1$ and $Q 2$ intercompartmental clearance, $R S E$ relative standard error, $V c$ volume of distribution of the central compartment, $V 2$ and $V 3$ volume of distribution of the peripheral compartments

a $\%$ RSE $=100 \% \times$ STD ERR/EST, STD ERR standard error, EST estimate

b $95 \% \mathrm{CI}=\mathrm{EST} \pm 1.96 \times \mathrm{STD}$ ERR, STD ERR standard error

c $\% \mathrm{CV}=100 \% \times \mathrm{SQRT}(\mathrm{EST})$ for $\operatorname{ETA}(\mathrm{p})$ and $\operatorname{EPS}(\mathrm{q}), S Q R T$ square root, ETA(p) refers to variance of Inter-individual variability, EPS(p) refers to Sigma (variance of residual error)

d Terminal half-life was calculated based on typical value of CL, Vc, V2, V3, Q1, and Q2 
used to produce the visual predictive check analysis and plotting.

\section{Results}

\subsection{Demographics and Baseline Characteristics}

A total of 57 subjects received at least one dose of dilmapimod, and 20 subjects were randomised to receive placebo in the four cohorts. A summary of the demographic variables and the baseline characteristics of the population is presented in Christie et al. [10].

Briefly, the median age across all subjects was 39 years. The median body weight and height were $86 \mathrm{~kg}$ and $175 \mathrm{~cm}$, respectively, with a median body mass index (BMI) of $27 \mathrm{~kg} / \mathrm{m}^{2}$. The median values for ISS total injury score and Glasgow Coma Score were 25 and 15, respectively, indicating severe/critical trauma and mild brain injury,
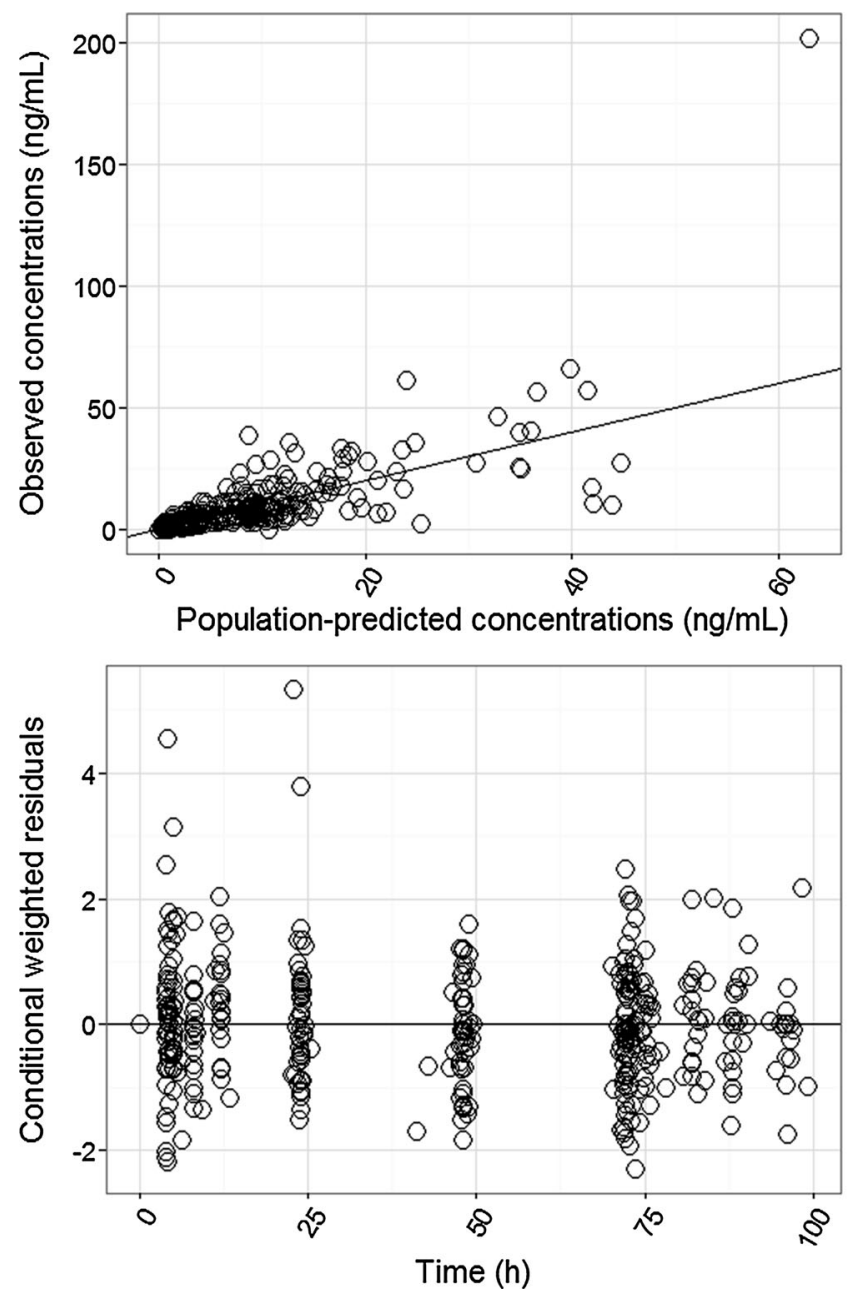

Fig. 2 Basic goodness-of-fit plots for the final dilmapimod pharmacokinetic model. Observed dilmapimod concentrations $(\mathrm{ng} / \mathrm{mL})$ are plotted vs. population and individual predictions (top). Conditional respectively. The median time since trauma was about $22 \mathrm{~h}$, with a range of 9-28 h. It was noted that only a small number of subjects were dosed in the early time post injury (four subjects were dosed within the first $12 \mathrm{~h}$ following injury, 41 were dosed between 12 and $24 \mathrm{~h}$ following injury, and 28 subjects were dosed between 24 and $36 \mathrm{~h}$ post injury).

Overall, there was no observable difference in demographics and baseline characteristics among the different treatment groups.

\subsection{Population PK Results}

The dilmapimod plasma concentration following intravenous infusion was adequately described by a threecompartment model with first-order elimination (left panel, Fig. 1). Table 2 shows the parameter estimates of the final PK model.

The fixed-effect parameters were estimated with good precision, with \%RSE ranging between 2.25 and $24.4 \%$.
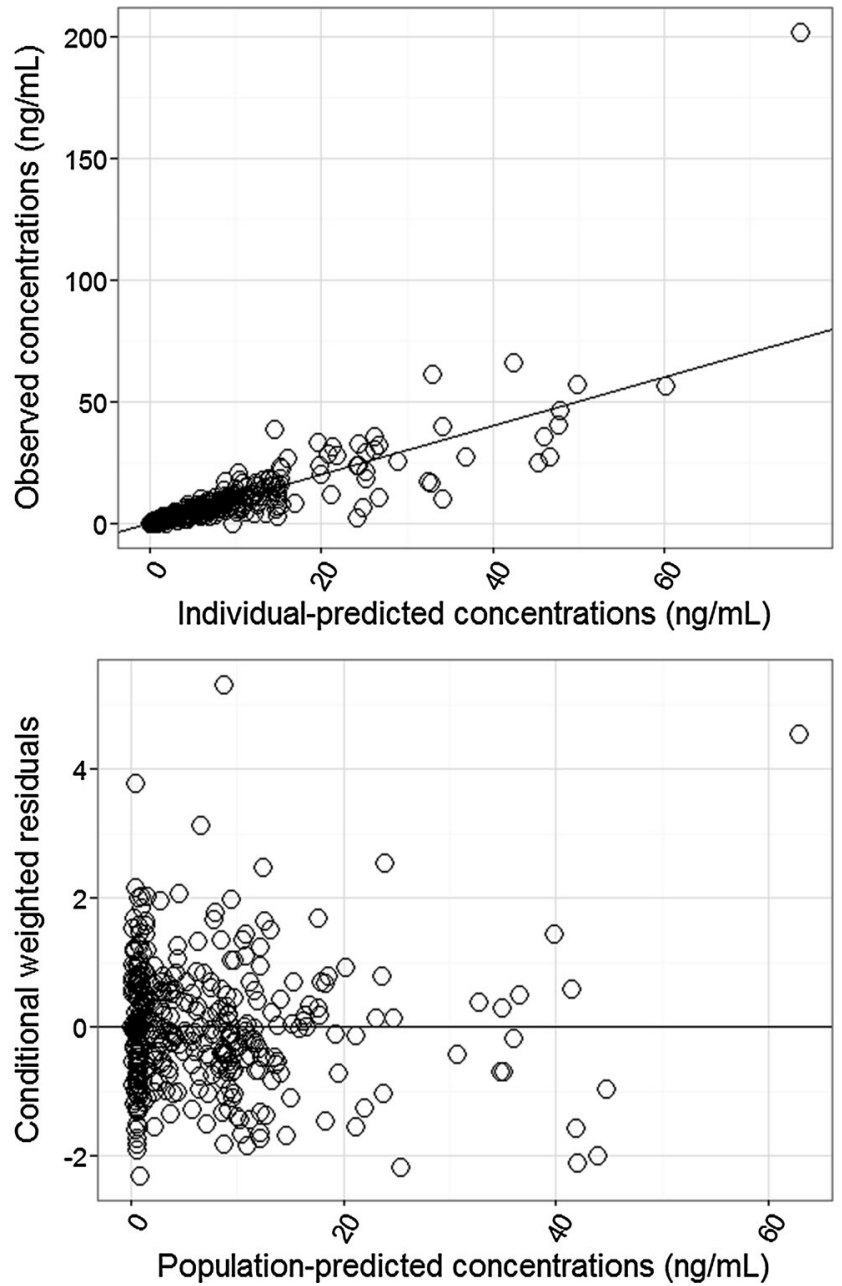

weighted residuals are plotted against time and population predictions (bottom). The empty circles represent the data points. The solid black line in each plot is the line of identity 
The inter-individual variability for $\mathrm{CL}$ and $\mathrm{Q} 2$ were moderate $(31.5 \%$ and $47.5 \%$, respectively). The effect of BMI on CL and Q2 was modelled using a power model with the covariate centred to the population mean of $27.4 \mathrm{~kg} / \mathrm{m}^{2}$. An increase in BMI of $1 \mathrm{~kg} / \mathrm{m}^{2}$ resulted in a $1.79 \mathrm{~L} / \mathrm{h}$ and $0.52 \mathrm{~L} / \mathrm{h}$ increase in CL and Q2, respectively.

The proportional error model was adequate, and the residual variability was about $38 \%$. The population clearance (CL) parameter value was $35.87 \mathrm{~L} / \mathrm{h}$, and the steadystate volume of distribution (Vss) (sum of Vc, V2, and V3) was $160 \mathrm{~L}$. A two-compartment model was tested and found not as good as a three-compartment model.

Standard model diagnostics and visual predictive check were conducted to assess the validity of the model. Figure 2 shows the goodness of fit for population predicted versus observed, individual predicted versus observed, conditional weighted residual versus time, and conditional weighted residuals versus population predicted concentrations. Figure 3 shows the visual predictive check for the final model.

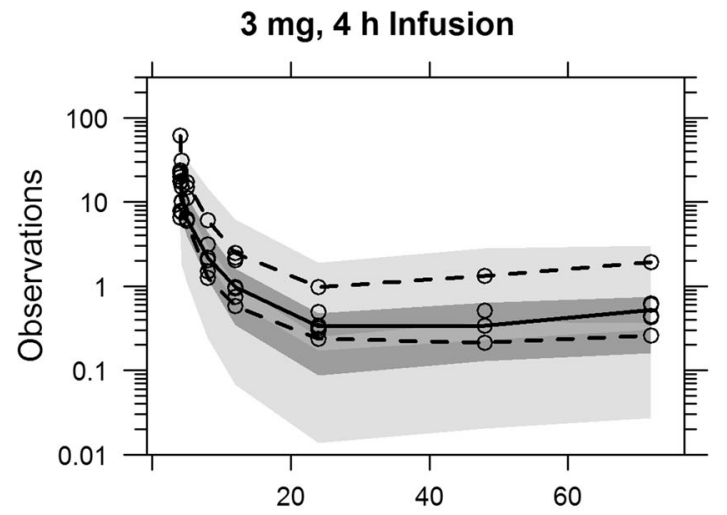

Time after dose

\section{$7.5 \mathrm{mg}, 4 \mathrm{~h}$ Infusion}

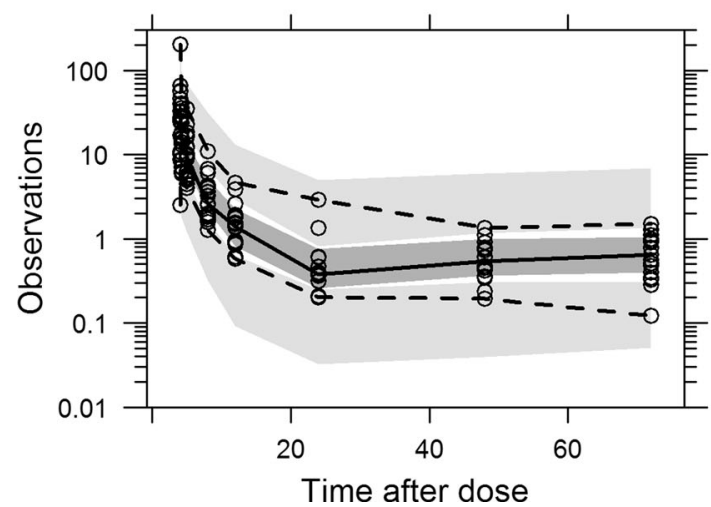

Fig. 3 Visual predicted check from 1000 simulated data sets for the final dilmapimod pharmacokinetic model. The $95 \%$ confidence interval (CI) of the median of the simulated data is represented by the dark grey area. The $95 \% \mathrm{CI}$ of the 2.5 th and 97.5 th percentiles of the simulated data are represented by the light grey areas. The
Dilmapimod plasma concentrations-time courses for each subject included in the analysis dataset were predicted from the final model, using their individual parameter and BMI values and individual dosing history. The predicted individual plasma concentrations at the sampling time where the PD data were collected were merged with the PD dataset for the following PK/PD analysis. The daily exposure $\mathrm{AUC}(0-24)$ and maximum concentration (Cmax), which were estimated from the final PK model for each individual in the dataset, are summarised by cohort and day. The results are presented in Christie et al. [10].

\subsection{CRP PK/PD Modelling Results}

A base model development was initiated to describe the time course of the systemic CRP levels without a drug effect included in the model, assuming that the CRP levels will quickly increase after injury, reach a peak and then gradually decrease (Eqs. 3, 4). The base model parameter estimates of fixed and random effects are presented in
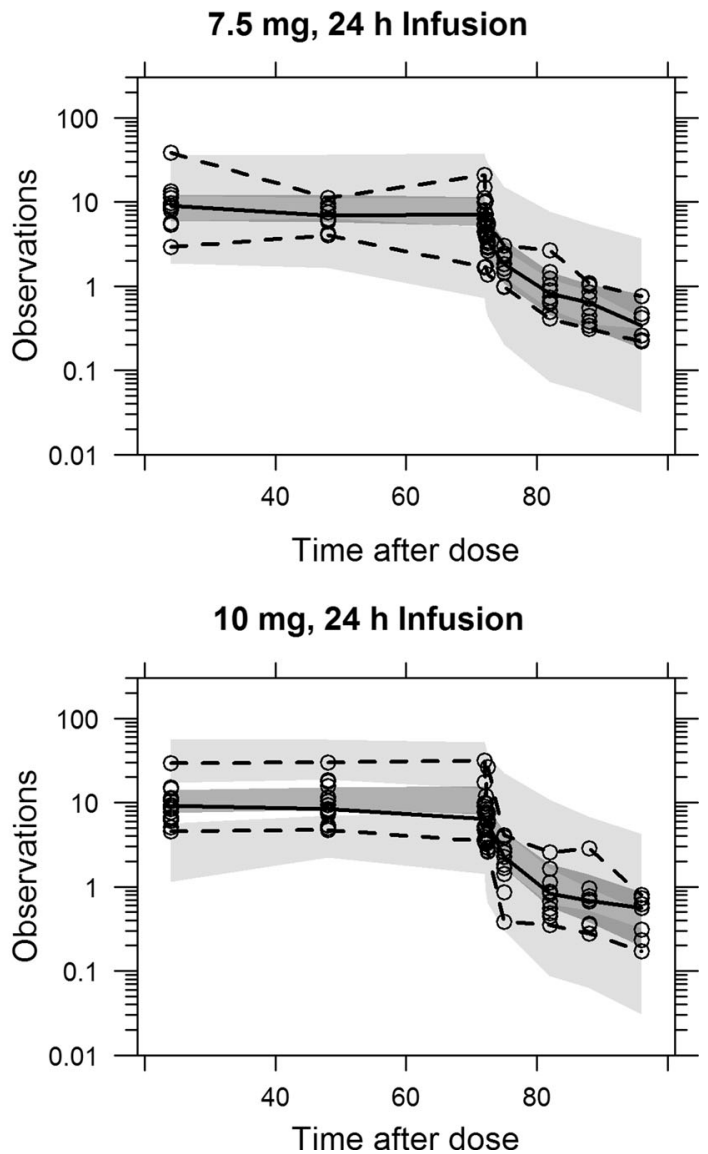

observed data are represented by the black open circles. The median of the observed data is represented by the solid line. The 2.5 th and 97.5th percentiles of the observed data are represented by the dashed lines 
Table 3 Parameter estimates of fixed and random effects from the base (no drug effect) CRP pharmacokineticl pharmacodynamic model

\begin{tabular}{|c|c|c|c|c|}
\hline Parameter (unit) & Typical value & $\% \mathrm{RSE}^{\mathrm{a}}$ & Standard error & $95 \% \mathrm{CI}^{\mathrm{b}}$ or $\% \mathrm{CV}^{\mathrm{c}}$ \\
\hline$K_{\text {decline }}\left(\mathrm{h}^{-1}\right)=\exp \left(\theta_{\text {pop }, 1}\right)$ & 0.008 & & & \\
\hline$\theta_{\text {pop }, 1}$ & -4.77 & -3.92 & 0.187 & -5.14 to -4.40 \\
\hline$K_{\mathrm{in}} 0\left(\mathrm{~h}^{-1}\right)=\exp \left(\theta_{\mathrm{pop}, 2}\right)$ & 7.171 & & & \\
\hline$\theta_{\mathrm{pop}, 2}$ & 1.97 & 3.17 & 0.0625 & 1.85 to 2.09 \\
\hline$K_{\text {out }}\left(\mathrm{h}^{-1}\right)=\exp \left(\theta_{\mathrm{pop}, 3}\right)$ & 0.026 & & & \\
\hline$\theta_{\text {pop }, 3}$ & -3.64 & -4.01 & 0.146 & -3.93 to -3.35 \\
\hline \multicolumn{5}{|l|}{ Inter-individual variability } \\
\hline$K_{\text {decline }}$ & 0.377 & 40.8 & 0.154 & 61.4 \\
\hline$K_{\text {in }} 0$ & 0.230 & 19.3 & 0.0444 & 48.0 \\
\hline$K_{\text {out }}$ & 0.373 & 29.5 & 0.110 & 61.1 \\
\hline \multicolumn{5}{|l|}{ Residual variability } \\
\hline Sigma & 0.216 & 3.44 & $7.44 \mathrm{e}-03$ & 46.5 \\
\hline
\end{tabular}

Shrinkage: $39.0 \%$ on $K_{\text {decline }}, 5.80 \%$ on $K_{\text {in }} 0,27.2 \%$ on $\mathrm{K}_{\text {out }}$, and $9.93 \%$ on Sigma

Condition number 9.91

$C I$ Confidence interval, CRP C-reactive protein, $C V$ coefficient of variation, $K_{\text {decline }}$ gradual decline in production rate over time, $K_{\text {in }} O \mathrm{CRP}$ production rate immediately following injury, $K_{\text {out }}$ first-order elimination rate constant of CRP, RSE relative standard error

a $\%$ RSE $=100 \% \times$ STD ERR/EST, STD ERR standard error, EST estimate

b $95 \% \mathrm{CI}=\mathrm{EST} \pm 1.96 \times \mathrm{STD}$ ERR, STD ERR standard error

c $\% \mathrm{CV}=100 \% \times \mathrm{SQRT}(\mathrm{EST})$ for ETA(p) and EPS(q), SQRT square root, ETA(p) refers to variance of Inter-individual variability, EPS(p) refers to Sigma (variance of residual error)

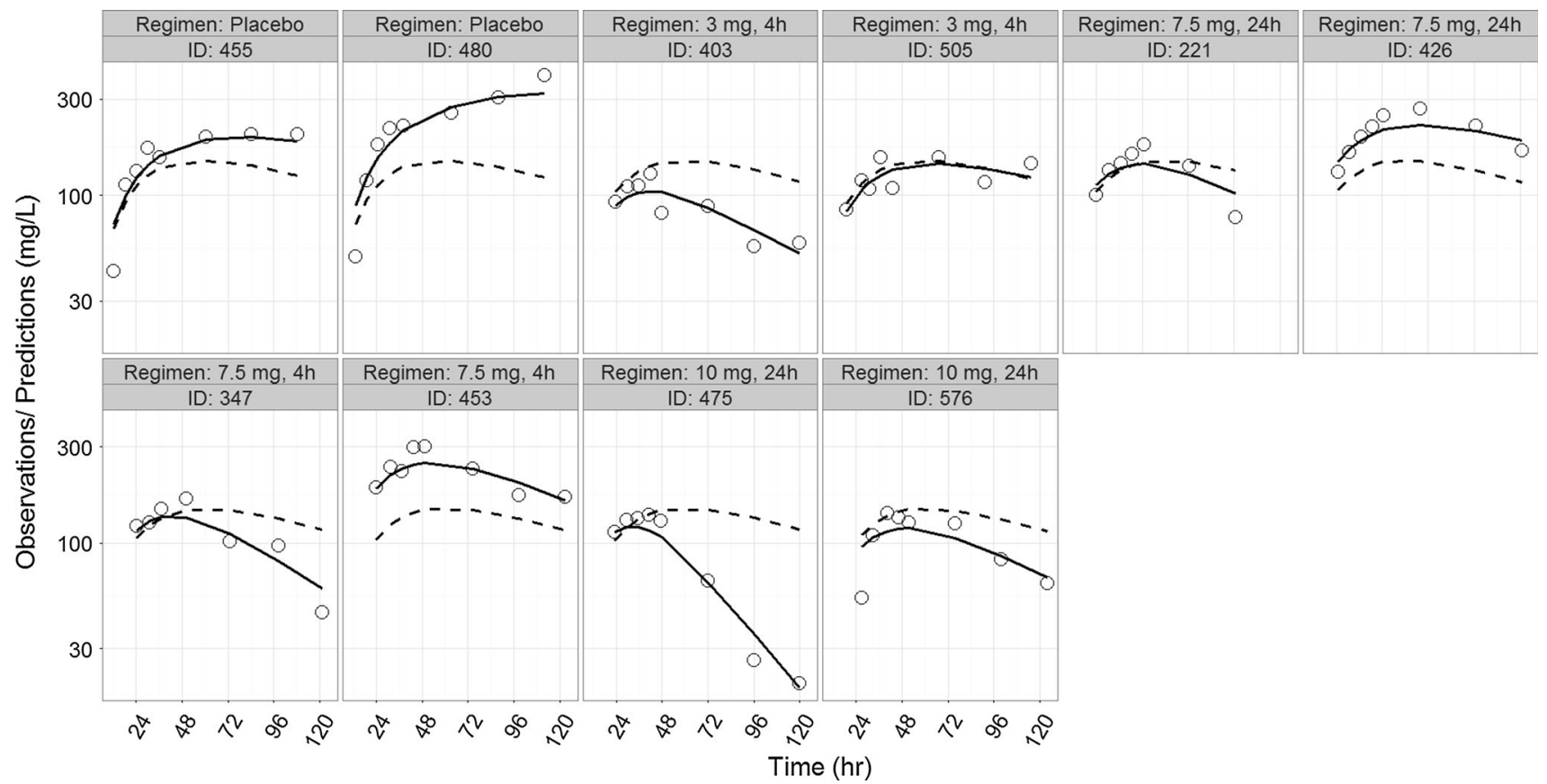

Fig. 4 Individual C-reactive protein (CRP) profiles from four representative subjects, two in each treatment group. The observed CRP concentrations are represented by the black open circles. The

Table 3. All fixed-effect parameters were estimated with good precision. Inter-individual variability was estimated for all parameters with moderate to relatively high precision (from $48 \%$ to $61 \%$ ). population and individual predictions are represented by the dashed and solid lines, respectively

Figure 4 shows the model predicted CRP profiles for four representative subjects, one in each treatment group, along with the observed data, and indicates that the model describes the shape of the systemic CRP time course 

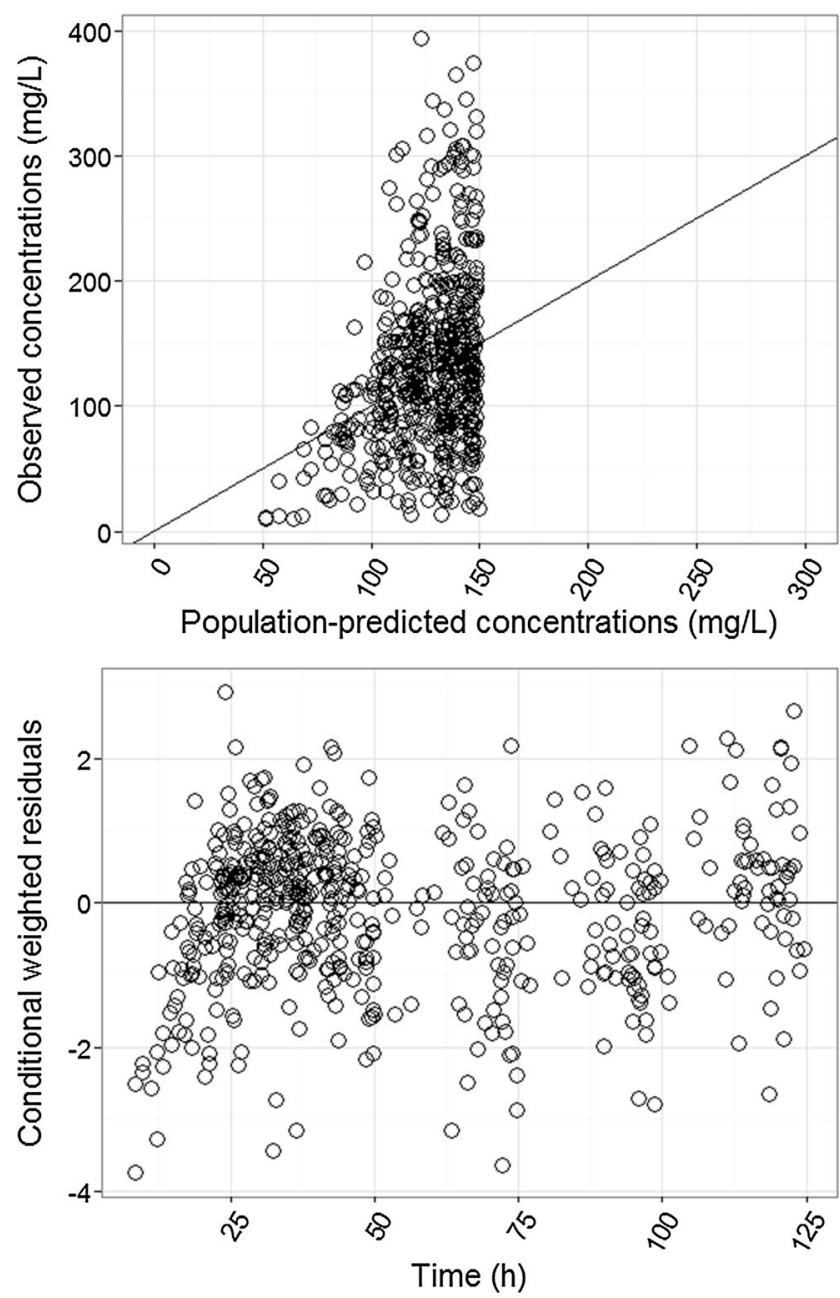

Fig. 5 Basic goodness-of-fit plots for the base C-reactive protein (CRP) model. Observed CRP concentrations $(\mathrm{mg} / \mathrm{L})$ are plotted vs. population and individual predictions (top). Conditional weighted

reasonably well. The goodness-of-fit plots (Fig. 5) did not show any obvious bias. The visual predictive check plots (Fig. 6) indicate reasonable fit of the model to the data, albeit the variability of the prediction was high.

An initial graphical analysis was conducted to explore the need for a dilmapimod drug effect term in the model. Examination of inter-subject variability (ETAs) versus treatment regimen (Fig. 7a) and versus dilmapimod exposure (AUC) (Fig. 7b) revealed potential drug effects on $K_{\text {decline }}$ (describing the gradual decline in production rate overtime), $K_{\text {in }} 0$, and on $K_{\text {out }}$ (first-order elimination rate constant of CRP).

\subsubsection{Drug-Effect Model}

Based on the current understanding of the $\mathrm{p} 38$ pathway, the potential association between systemic exposure of dilmapimod and CRP biomarker was investigated. Thus, the effect of dilmapimod on CRP was first assessed using
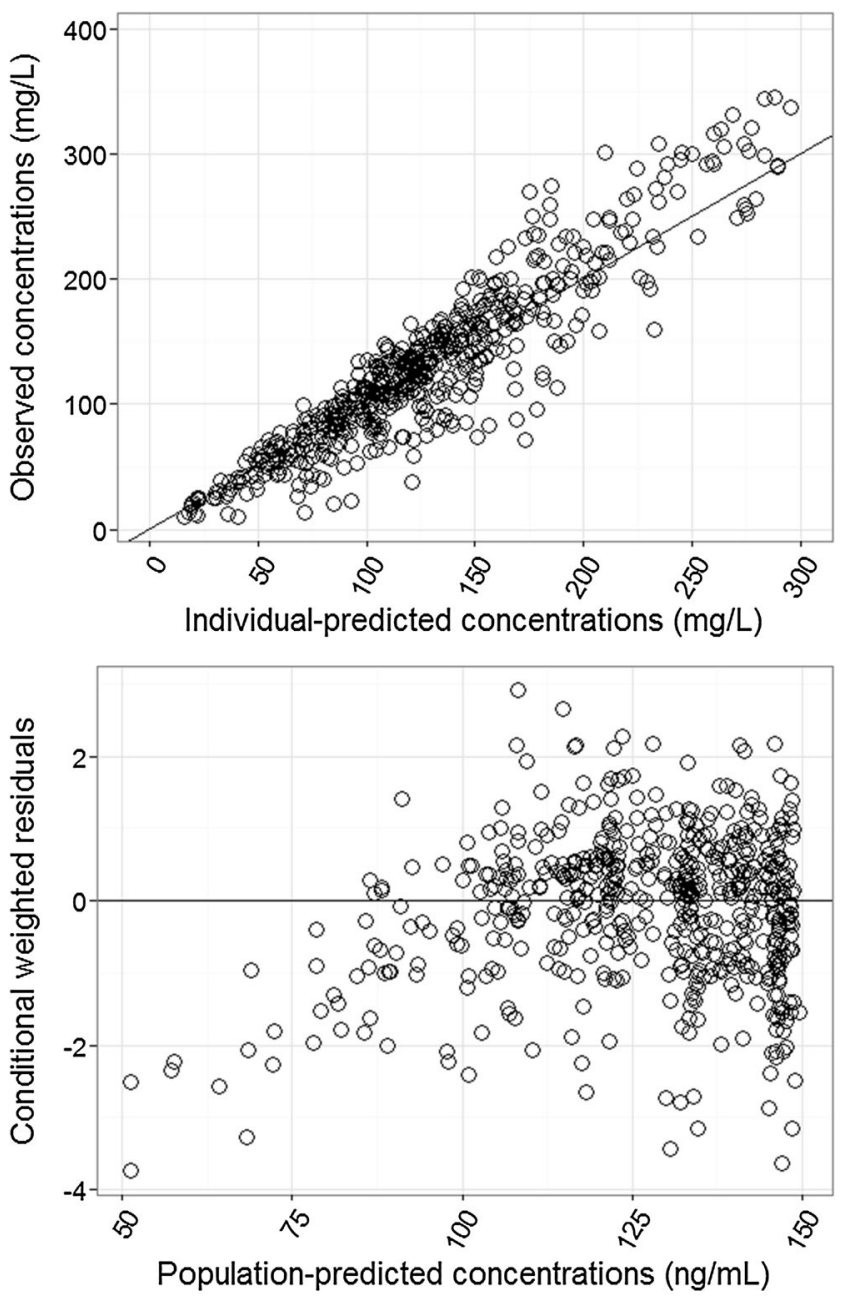

residuals are plotted against time and population predictions (bottom). The empty circles represent the data points. The solid line in each plot is the line of identity

inhibitory models, assuming that inhibition of the p38 pathway by dilmapimod would result in an inhibition of the production rate of CRP (Table 1). However, this modelling approach did not result in an acceptable drug-effect model (data not shown). Furthermore, based on the graphical analysis presented in Fig. 7, additional stimulatory mechanistic drug-effect models on $K_{\text {decline }}$ and $K_{\text {out }}$ were explored (Table 1). None of these models resulted in an acceptable drug-effect model for the dataset under evaluation (data not shown).

\section{Discussion}

In this work, we present a population PK and PK/PD approach to assist the understanding of PK and PD profiles following intravenous infusion of dilmapimod.

The plasma concentration data of dilmapimod obtained in this study were best described by a three-compartment 
Fig. 6 Visual predicted check from 1000 simulated data sets for the base $\mathrm{C}$-reactive protein (CRP) model. The 95\% confidence interval (CI) of the median of the simulated data is represented by the dark grey area. The $95 \%$ CI of the 2.5 th and 97.5 th percentiles of the simulated data are represented by the light grey areas. The observed data are represented by the black open circles. The median of the observed data is represented by the solid line. The 2.5 th and 97.5 th percentiles of the observed data are represented by the dashed lines

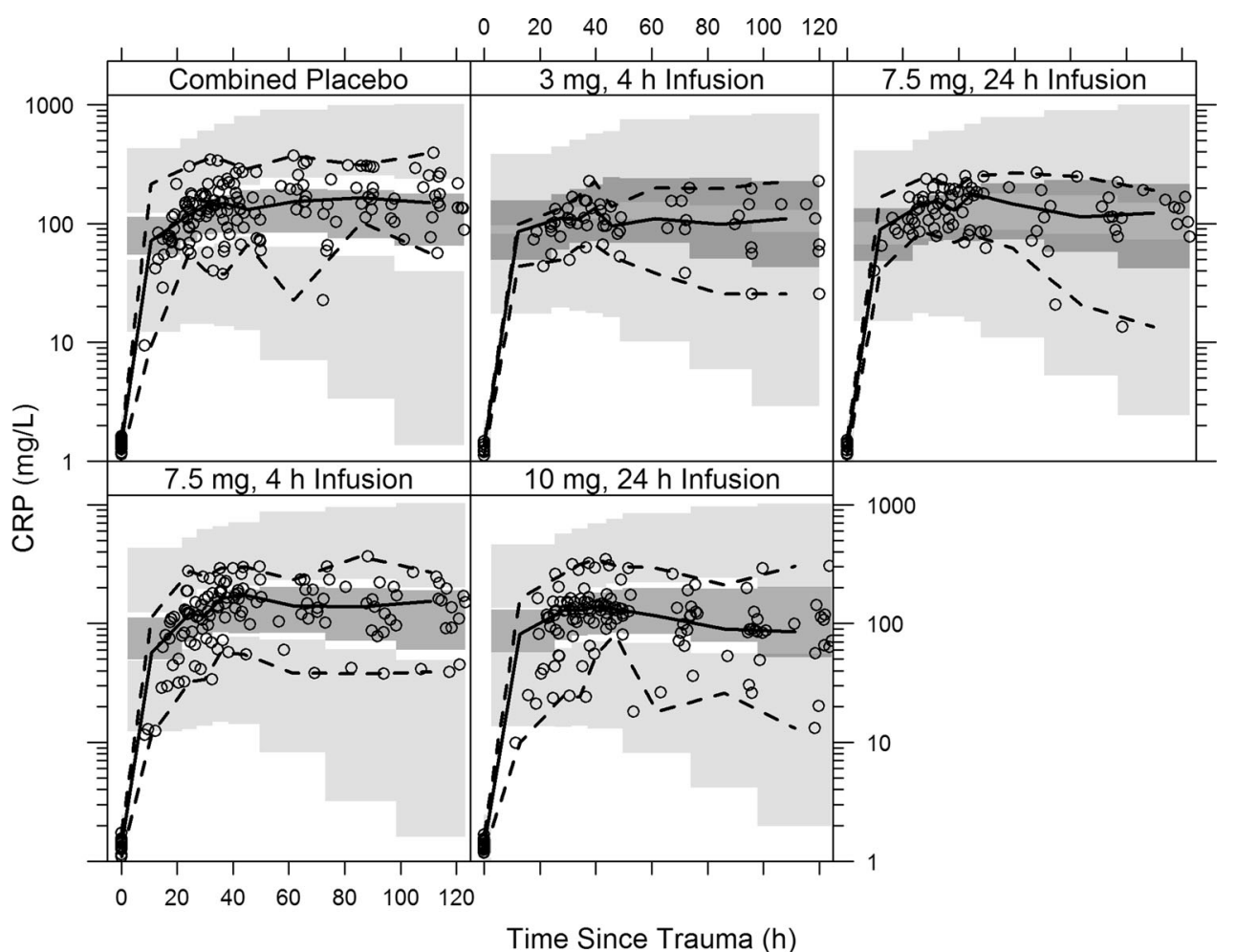

model, with inter-individual variability added on clearance and inter-compartment clearance Q2. Data did not support adding inter-individual variability on other PK parameters. Among all the demographic and baseline covariates, BMI was found to be a statistically significant covariate on CL and Q2. Based on the final population PK model, the clearance for dilmapimod for the typical patient (mean BMI of $27.4 \mathrm{~kg} / \mathrm{m}^{2}$ ) in the current study was $36 \mathrm{~L} / \mathrm{h}$. The clearance was about $21 \%$ lower and $23 \%$ higher for patients with BMI of $23 \mathrm{~kg} / \mathrm{m}^{2}$ [mean - standard deviation (SD)] and $32 \mathrm{~kg} / \mathrm{m}^{2}$ (mean $+\mathrm{SD}$ ) compared with the typical patients, respectively. The BMI effect on dilmapimod clearance is not completely unexpected as a slight correlation of BMI with oral clearance in healthy individuals and chronic obstructive pulmonary disease (COPD) patients has been previously observed (data not shown). This correlation may be explained by the fact that dilmapimod is eliminated almost exclusively by metabolism (hydroxylation and glucuronidation as the primary routes of metabolism), and obesity was shown to increase both phase I and phase II metabolism of various medications [16-19]. However, the changes in clearance and therefore total systemic exposure (dose/CL) were not considered clinically significant; therefore, dose adjustments were not considered necessary at this stage.

Exploratory analyses conducted to assess the impact of dilmapimod on CRP showed a trend of lower levels of CRP present at later time points (e.g. $72 \mathrm{~h}$ post dose) at all dilmapimod dose levels, relative to placebo [10]. The decrease in CRP levels was most apparent with the continuous infusion of $10 \mathrm{mg} / 24 \mathrm{~h} \mathrm{[10]}$.

The potential association between the systemic exposure of dilmapimod and CRP levels was explored using mechanistic modelling. An initial base model described the systemic CRP levels without drug effect based on the assumptions that after injury, CRP levels quickly increase, reach a peak and then gradually decrease. Although the time of injury was known for patients with trauma, there was no actual measurement of CRP until the subject was randomised in the study. Therefore, the baseline CRP level was set at the normal level for CRP. Even though the base model overpredicts the CRP variability, this approach is considered appropriate as these subjects were generally healthy before the injury. The overprediction of CRP variability may be due to the high variability in the timing of treatment initiation relative to the time of trauma (ranging between 4 and $36 \mathrm{~h}$ ), or due to the severity and type of injury.

Exploration of individual parameters $K_{\mathrm{in}} 0, K_{\text {decline }}$, and $K_{\text {out }}$ by treatment group, and systemic exposure of dilmapimod (AUC) indicated a potential effect of dilmapimod on reduction of $K_{\mathrm{in}} 0$ or stimulation of $K_{\text {decline }}$ and $K_{\text {out }}$. However, when the effect of dilmapimod on the CRP level was investigated, the indirect response modelling approaches did not result in an acceptable drug-effect model. This could be due to the high variability observed in CRP levels, or the relatively narrow dose and systemic exposure ranges. Similar a PK/PD modelling 

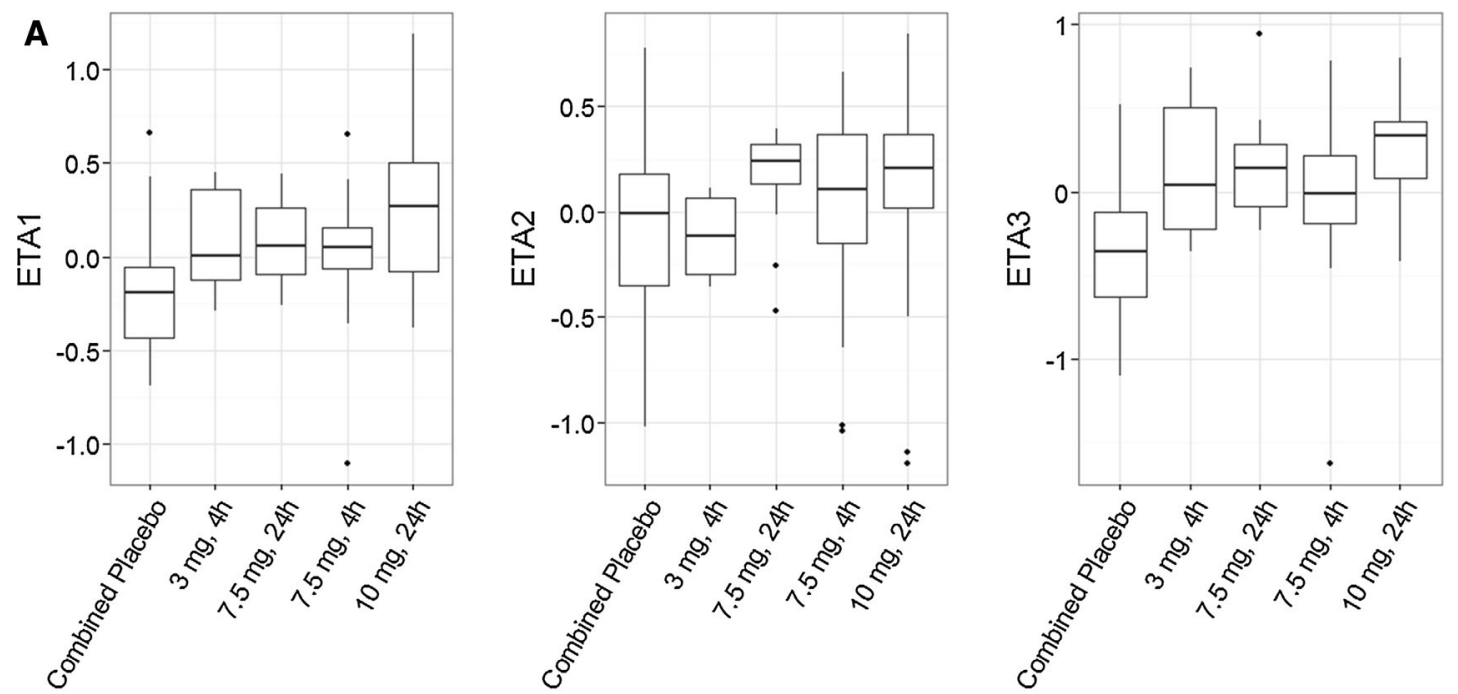

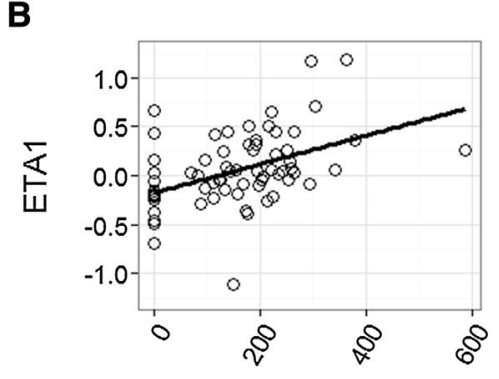

AUC24 (hr*ng/mL)

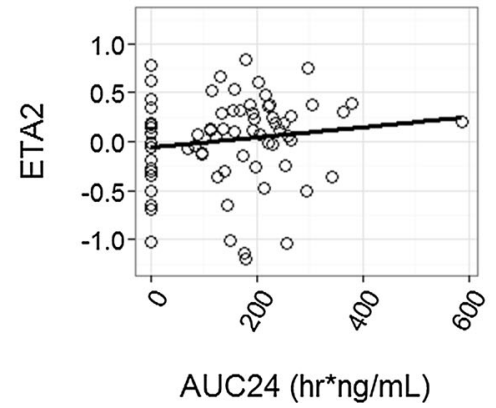

Fig. 7 Box plot of estimated random effects on $K_{\text {decline }}$ (ETA1), $K_{\text {in }} 0$ (ETA2), and $K_{\text {out }}$ (ETA3) by treatment groups for the base C-reactive protein (CRP) model (a). Linear regression plots of the estimated random effects vs. AUC (ng.h/mL) (b). The empty circles represent

approach was also conducted on IL-6 data in this study [20]. However, the overall effect of dilmapimod on IL-6 levels was not revealed via such a modelling frame, in spite of some observed effect of dilmapimod at some time points [10].

Dilmapimod intravenous infusion up to $10 \mathrm{mg}$ was generally well tolerated in patients with severe trauma at risk of ARDS. Evidence of hepatotoxicity has been associated with some p38 MAPK inhibitors [21, 22], which led to cautious selection of dilmapimod's top dose in this study.

Another important factor for the ability to develop a mechanistic drug-effect model for dilmapimod may be the time of treatment start. Transcription of CRP is regulated by pro-inflammatory cytokines, including IL-6 [23, 24], and therefore, a reduction in IL-6 should precede a decrease in CRP. Indeed, evaluation of serum IL-6 and CRP fluctuation in children with severe head injury showed serum IL- 6 values peaked $4 \mathrm{~h}$ post injury, followed by a decrease over time, while the CRP levels reached peak levels between 24 and $48 \mathrm{~h}$, decreasing thereafter [22]. In this study, only four subjects were dosed within $12 \mathrm{~h}$ post the data points. The solid lines represent linear regression lines. AUC area under the concentration-time curve, $K_{\text {decline }}$ decline in CRP production rate over time, $K_{\text {in }} O \mathrm{CRP}$ production rate immediately following injury, $K_{\text {out }}$ first-order elimination rate constant of CRP

injury, 41 subjects were dosed between 12 and $24 \mathrm{~h}$ post injury, and 28 subjects were dosed after $24 \mathrm{~h}$. Given the role of IL-6 in transcriptional regulation of CRP and the long half-life of CRP (measured in days), it is possible that administration of the p38 MAPK inhibitor dilmapimod shortly after injury would result in a more robust prevention of injury-induced elevation in CRP levels [24].

Exploratory analyses conducted to assess the impact of dilmapimod on IL-6 showed a trend of lower levels of IL-6 present $24 \mathrm{~h}$ post dose at all dilmapimod dose levels, relative to placebo [10]. The continuous infusion of $10 \mathrm{mg} /$ $24 \mathrm{~h}$ was the only dose level that yielded ratios of IL-6 relative to placebo below 1 up to $72 \mathrm{~h}$ post dose [10]. Consistent with the role of IL-6 in the transcriptional regulation of CRP, the decrease in IL-6 following dilmapimod administration preceded the decrease in CRP [10]. Therefore, an alternative modelling strategy could be a modified indirect response model that describes the stimulatory effect of IL-6 on the production rate of CRP, with dilmapimod having indirect inhibitory activity on IL-6 production. 


\section{Conclusions}

Dilmapimod plasma concentration-time profiles in severe trauma subjects at risk for ARDS were described by a three-compartment model. Following intravenous dosing, dilmapimod was quickly distributed to peripheral compartments and then slowly eliminated in a multi-exponential manner. The plasma concentration of dilmapimod increased approximately proportionally to the increase in dose. BMI was found to be a significant covariate for CL and Q2.

The CRP profile post injury was adequately described by an indirect response model, with a sharp increase in the CRP levels following injury, then them slowly diminishing. Although data exploration indicated potential drug effects of dilmapimod inhibiting the production of CRP levels, the current small dataset did not show a statistically significant improvement in the PK/PD modelling.

Acknowledgements The authors thank all members of the project team, in particular, Aili Lazaar, David Lipson, Andrew Bayliffe, Will Powley, Simon Parry, Kelly Hardes, and Lesley Kahl for their support in the design and execution of this study.

\section{Compliance with ethical standards}

Conflict of interest Shuying Yang, and Teodora Pene Dumitrescu are employees of GSK and hold GSK stock.

Funding This study was funded by GlaxoSmithKline (GSK study AL1111592; NCT00996840).

Ethical standards Local ethics review committee/institutional review board approvals were obtained, and the study was conducted in accordance with Good Clinical Practice guidelines and the Declaration of Helsinki 2008. Written, informed consent was obtained from each patient before any study procedure was performed.

Open Access This article is distributed under the terms of the Creative Commons Attribution-NonCommercial 4.0 International License (http://creativecommons.org/licenses/by-nc/4.0/), which permits any noncommercial use, distribution, and reproduction in any medium, provided you give appropriate credit to the original author(s) and the source, provide a link to the Creative Commons license, and indicate if changes were made.

\section{References}

1. Han S, Mallampalli RK. The acute respiratory distress syndrome: from mechanism to translation. J Immunol. 2015;194(3):855-60.

2. Matthay MA, Ware LB, Zimmerman GA. The acute respiratory distress syndrome. J Clin Invest. 2012;122(8):2731-40.

3. Ware LB, Matthay MA. The acute respiratory distress syndrome. N Engl J Med. 2000;342(18):1334-49.

4. Anand P, Shenoy R, Palmer JE, Baines AJ, Lai RY, Robertson J, et al. Clinical trial of the p38 MAP kinase inhibitor dilmapimod in neuropathic pain following nerve injury. Eur $J$ Pain. 2011;15(10):1040-8.
5. Lukey P, Perry H, Yang S, Parry S, Dickson M, Norris V, et al. Single doses of p38 MAP kinase inhibitors or prednisolone affect CRP and IL-6 in patients with active rheumatoid arthritis (RA). Open J Immunol. 2012;2(3):85-97.

6. Sarov-Blat L, Morgan JM, Fernandez P, James R, Fang Z, Hurle $\mathrm{MR}$, et al. Inhibition of $\mathrm{p} 38$ mitogen-activated protein kinase reduces inflammation after coronary vascular injury in humans. Arterioscler Thromb Vasc Biol. 2010;30(11):2256-63.

7. Singh D, Smyth L, Borrill Z, Sweeney L, Tal-Singer R. A randomized, placebo-controlled study of the effects of the p38 MAPK inhibitor SB-681323 on blood biomarkers of inflammation in COPD patients. J Clin Pharmacol. 2010;50(1):94-100.

8. Liu S, Feng G, Wang GL, Liu GJ. p38MAPK inhibition attenuates LPS-induced acute lung injury involvement of NF-kappaB pathway. Eur J Pharmacol. 2008;584(1):159-65.

9. Ma J, Ouyang B, Lai JB, Guan XD. Engagement of p38 mitogenactivated protein kinase in regulation of epithelial barrier of acute respiratory distress syndrome. Zhonghua Wei Zhong Bing Ji Jiu Yi Xue. 2013;25(10):589-93.

10. Christie JD, Vaslef S, Chang PK, May AK, Gunn SR, Yang S, et al. A randomized dose-escalation study of the safety and antiinflammatory activity of the p38 mitogen-activated protein kinase inhibitor dilmapimod in severe trauma subjects at risk for acute respiratory distress syndrome. Crit Care Med. 2015;43(9):1859-69.

11. Yudkin JS, Stehouwer CD, Emeis JJ, Coppack SW. C-reactive protein in healthy subjects: associations with obesity, insulin resistance, and endothelial dysfunction: a potential role for cytokines originating from adipose tissue? Arterioscler Thromb Vasc Biol. 1999;19(4):972-8.

12. Giannoudis PV, Hildebrand F, Pape HC. Inflammatory serum markers in patients with multiple trauma. Can they predict outcome? J Bone Joint Surg Br. 2004;86(3):313-23.

13. Giannoudis PV, Smith MR, Evans RT, Bellamy MC, Guillou PJ. Serum CRP and IL-6 levels after trauma. Not predictive of septic complications in 31 patients. Acta Orthop Scand. 1998;69(2):184-8.

14. Gosling P, Dickson GR. Serum c-reactive protein in patients with serious trauma. Injury. 1992;23(7):483-6.

15. Kalabalikis P, Papazoglou K, Gouriotis D, Papadopoulos N, Kardara M, Papageorgiou F, et al. Correlation between serum IL6 and CRP levels and severity of head injury in children. Intensive Care Med. 1999;25(3):288-92.

16. Abernethy DR, Greenblatt DJ, Divoll M, Shader RI. Enhanced glucuronide conjugation of drugs in obesity: studies of lorazepam, oxazepam, and acetaminophen. J Lab Clin Med. 1983;101(6):873-80.

17. Brill MJ, Diepstraten J, van RA, van KS, van den Anker JN, Knibbe CA. Impact of obesity on drug metabolism and elimination in adults and children. Clin Pharmacokinet. 2012;51(5):277-304.

18. Marik P, Varon J. The obese patient in the ICU. Chest. 1998;113(2):492-8.

19. Morgan DJ, Bray KM. Lean body mass as a predictor of drug dosage. Implications for drug therapy. Clin Pharmacokinet. 1994;26(4):292-307.

20. GSK. Clinical Pharmacology Study Report: Assessment of the anti-inflammatory activity, efficacy and safety of Intravenous SB681323 in subjects at risk for development of acute lung injury (ALI) or acute respiratory distress syndrome (ARDS). 2015. https://wwwgsk-clinicalstudyregistercom/study/ 111592? search=compound\&compound=dilmapimod\#csr

21. Genovese MC, Cohen SB, Wofsy D, Weinblatt ME, Firestein GS, Brahn E, et al. A 24-week, randomized, double-blind, placebocontrolled, parallel group study of the efficacy of oral SCIO-469, 
a p38 mitogen-activated protein kinase inhibitor, in patients with active rheumatoid arthritis. J Rheumatol. 2011;38(5):846-54.

22. Schreiber S, Feagan B, D'Haens G, Colombel JF, Geboes K, Yurcov $\mathrm{M}$, et al. Oral p38 mitogen-activated protein kinase inhibition with BIRB 796 for active Crohn's disease: a randomized, double-blind, placebo-controlled trial. Clin Gastroenterol Hepatol. 2006;4(3):325-34.
23. Eklund CM. Proinflammatory cytokines in CRP baseline regulation. Adv Clin Chem. 2009;48:111-36.

24. Pepys MB, Hirschfield GM. C-reactive protein: a critical update. J Clin Invest. 2003;111(12):1805-12. 